A N N A L E S Annales de Bretagne et des Pays de l'Ouest

Anjou. Maine. Poitou-Charente. Touraine

$115-4 \mid 2008$

Varia

\title{
Le théâtre municipal de Cholet
}

Misère et noblesse d'un équipement culturel de province dans l'entredeux-guerres

\section{François Drémeaux}

\section{(2) OpenEdition}

\section{Journals}

Édition électronique

URL : http://journals.openedition.org/abpo/226

DOI : 10.4000/abpo.226

ISBN : 978-2-7535-1512-3

ISSN : 2108-6443

Éditeur

Presses universitaires de Rennes

Édition imprimée

Date de publication : 31 décembre 2008

Pagination : 145-172

ISBN : 978-2-7535-0808-8

ISSN : 0399-0826

\section{Référence électronique}

François Drémeaux, «Le théâtre municipal de Cholet », Annales de Bretagne et des Pays de l'Ouest [En ligne], 115-4 | 2008, mis en ligne le 31 décembre 2010, consulté le 01 mai 2019. URL : http:// journals.openedition.org/abpo/226 ; DOI : 10.4000/abpo.226 


\title{
Le théâtre municipal de Cholet \\ Misère et noblesse d'un équipement culturel de province dans l'entre-deux-guerres
}

\author{
François DRÉMEAUX \\ Professeur d'histoire, \\ lycée français Victor-Segalen de Hong-Kong
}

La ville de Cholet semble construite autour de son théâtre municipal. Il trône devant la place Travot, centre de la capitale des Mauges. C'est un bâtiment néo-classique, récemment restauré, dont la clarté tranche sur les gris de l'esplanade, de l'église Notre-Dame et de la Poste d'influence Art Déco. Dans cette cité au passé architectural relativement pauvre, c'est un monument important d'histoire et de patrimoine. Il a connu les grandes heures des tournées théâtrales mais aussi les crises de ce milieu artistique, les pleurs et les rires de plusieurs générations. Il a subi les doutes des politiques culturelles de la ville et l'attention d'une communauté passionnée des arts de la scène. La période de l'entre-deux-guerres permet de mettre en valeur, à titre d'exemple, le rôle culturel mais aussi social d'un tel édifice dans une petite ville ouvrière de province ${ }^{1}$. L'époque est également un observatoire de l'organisation des représentations, du répertoire choisi, de la fréquentation ou encore de l'administration de cet équipement culturel de prestige ${ }^{2}$.

\section{Après la guerre, une lente réhabilitation}

Le 23 janvier 1884, la construction d'un théâtre est décidée par le conseil municipal. Après deux ans de travaux, le bâtiment est inauguré le 5 octobre

1. Avec une population de 19542 habitants en 1921 et 23385 habitants en 1936, Cholet est la deuxième ville de Maine-et-Loire. Située en pleine région d'élevage, son marché aux bœufs est actif mais c'est surtout ses industries textiles qui la rendent célèbre. C'est une ville ouvrière qui, jusqu'en 1932, donne ses suffrages à des élus radicaux-socialistes ou républicains de gauche avant d'élire, pour le reste de la période, un maire conservateur mais soucieux des équilibres sociaux.

2. Cet article est le résultat de recherches effectuées dans le cadre de notre maîtrise d'Histoire sur La vie culturelle et artistique à Cholet dans l'entre-deux-guerres, soutenue en 2002 à l'université d'Angers sous la direction de M. Yves Denéchère. 
1886. Ses dimensions extérieures sont de 30 mètres de long pour 15 mètres de large et la scène mesure 9 mètres sur 4,4 mètres. Sa capacité d'accueil est de 517 places dans la salle, à quoi il faut ajouter le foyer du théâtre prévu pour les réunions d'associations. À l'époque qui nous intéresse, l'acoustique est jugée " moyenne ${ }^{3}$ ".

Jusque dans les années 1920, quasiment aucune modification n'est apportée à l'édifice. Les 517 places sont réparties comme suit : 28 en loges, 80 fauteuils, 285 stalles et chaises, 24 en balcons, 100 en galeries. D'après les descriptions, la décoration semble assez riche :

" Nous devons à M. Biron ${ }^{4}$ les très belles sculptures extérieures et intérieures [...] Des chapiteaux, avec masques de comédie, complétaient ce remarquable ensemble. $M$. Alexandre Jouitteau exécuta, dans un style très XVIII , les dix panneaux des volets intérieurs, peints avec beaucoup de grâce. Enfin, M. Roustel brossa le rideau et les nombreux décors [...]. Quant au plafond de la salle, représentant la Musique et la Danse, le Drame et la Comédie, il était l'œuvre de M. Audfray de Paris ${ }^{5}$."

Par sa localisation et ses ornementations, le théâtre municipal fait bonne figure. Au sortir de la Grande Guerre, l'édifice est déjà le centre géographique de la ville et donne l'impression d'une cité dotée d'une dynamique culturelle.

Toutefois, l'ensemble est en mauvais état. « Les bâtiments communaux ont tant souffert de l'impossibilité de les entretenir pendant la guerre, que leur état est loin d'être satisfaisant ", souligne le maire Alexis Guérineau, lors du conseil municipal du 24 novembre 1923. Cinq ans après l'arrêt des combats, les traces de la guerre sont encore présentes. Certes, loin du front, aucun obus n'est tombé sur la ville; mais la priorité étant à l'effort de guerre, tout entretien a cessé pendant les quatre années d'hostilités, causant indirectement des dommages irréversibles. Pour preuve, cet incident relaté par l'hebdomadaire L'Intérêt Public en septembre 1919, lors d'une représentation de Faust : "Pendant le premier entracte, une moulure décorative en plâtre s'est détachée de la partie droite de la muraille et, tombant d'une grande hauteur, aurait pu causer un très grave accident, si elle n'avait d'abord atteint une balustrade sur laquelle elle s'est brisée, recouvrant de ses débris les spectateurs, mais heureusement sans les blesser. " Manque de crédits, autres priorités... les travaux pour prévenir ce genre d'incident tardent. Une note de service de l'architecte voyer reconnaît « l'absolue nécessité, en raison de l'état actuel des corniches (architraves et consoles) du plafond du théâtre, de condamner les places indiquées en plus de

3. Arch. mun. de Cholet, Correspondance du maire, 19 septembre 1936. Réponse au préfet relative à l'enquête " en vue d'établir un tableau général des ressources théâtrales".

4. François-Stanislas Biron est un sculpteur choletais renommé. Il meurt en 1926 à l'âge de 78 ans.

5. JEANNEAU, Pierre, Le théâtre de Cholet..., p. 17. Le théâtre ayant brûlé en 1949, il ne reste plus qu'une photographie du plafond de l'artiste Audfray. L'intérieur a été remanié par la suite. 
la première rangée de places dans toute la profondeur de la salle... ". Piètre solution qui retarde les réparations tandis que l'état de l'édifice ne s'arrange guère. En août 1922, divers aménagements sont réalisés et le chauffage est réparé suite au vote d'un crédit. En raison de la somme que sa réparation représente, il n'est toujours rien prévu pour le toit. Il faut attendre 1925, pour qu'un crédit de 50000 francs soit débloqué. Malgré « le prix très élevé du remaniement de la couverture [...] le conseil municipal reconnaît l'urgence des réparations à effectuer ${ }^{6}$ ".

Le premier aménagement consiste, en 1922, à redonner au théâtre municipal sa fonction d'origine. "La salle, organisée pour y donner des représentations cinématographiques n'a plus sa raison d'être, et il y a lieu de remettre le théâtre dans l'état primitif ${ }^{7}$. " C'est important puisque, selon le rapport Debureau, " nombre de gens attendent que ce travail soit fait pour fréquenter plus assidûment notre théâtre ». Le fonctionnement des représentations doit par ailleurs être assuré et les décors sont, à ce titre, primordiaux. "L'état de vétusté dans lequel se trouve une partie des décors du théâtre, a amené l'administration municipale à penser qu'il serait opportun de profiter du séjour à Cholet de monsieur Essers, peintre décorateur, pour faire procéder à la réfection de quelques décors ${ }^{8}$. " Le vote d'un crédit de 3400 francs n'est accepté qu'après d'âpres discussions.

\section{Les combats se poursuivent... sur scène}

Pendant et après la Grande Guerre, le comité dit « des œuvres de guerre " exploite un cinéma dans le théâtre municipal, avec une programmation classique faite d'actualités, d'intermèdes comiques et de films, français ou américains. Les bénéfices ont des destinations assez variées après l'armistice : à des cuvres de bienfaisance diverses ou au financement du monument aux morts ${ }^{9}$.

Le passage de troupes militaires pour des représentations musicales ou théâtrales est également chose courante. Le $313^{\mathrm{e}}$ Régiment américain est en première ligne de ce front culturel. Les soldats d'outre-atlantique proposent dans les premiers mois de 1919 quatre grands rendez-vous. Les dimanches 4 et 11 mai, au jardin du Mail pour des concerts, ainsi que le jeudi 20 mars et le mercredi 7 mai au théâtre pour des spectacles de variétés : «Un groupe de nos amis et alliés de l'armée américaine vient [...] donner une soirée au théâtre de notre ville : orchestre, chant, monologues, intermèdes comiques de leur pays ${ }^{10} \ldots$.. L'accueil du public est chaleureux : il s'agit du premier

6. Arch. mun. de Cholet. Délibérations du conseil municipal, 11 août 1925.

7. Arch. mun. de Cholet. Dossiers préparatoires du conseil municipal, 5 février 1923, rapport Debureau "sur la nécessité de remettre en état d'avant-guerre les places du théâtre municipal ".

8. Arch. mun. de Cholet. Délibérations du conseil municipal, 28 décembre 1923.

9. L'Intérêt Public, 5 janvier 1919. " Le cinéma des œuvres de guerre offre 5000 francs sur sa recette pour financer le monument aux morts de la Grande guerre de Cholet ".

10. L'Intérêt Public, 16 mars 1919. 
spectacle " vivant " au théâtre municipal depuis plus de quatre ans! Les soldats français brûlent également les planches du théâtre municipal dans l'année 1919. Avant le retour des militaires à la caserne Tharreau, le dépôt du $77^{\mathrm{e}}$ Régiment d'Infanterie donne deux soirées de gala, les mercredi 6 et jeudi 7 août 1919. Des divertissements en tout genre sont proposés et une grande partie de ces soirées est réservée à l'hommage aux morts et à la célébration patriotique. Le chroniqueur de L'Intérêt Public rend compte, entre autres, des chansons interprétées lors de ces soirées : "Verdun, on ne passe pas! ", "Le retour ", " Ode à nos morts ", etc.

Les anciens combattants ont priorité dans les démarches de réservation des salles de spectacle. L'Union nationale des combattants (UNC) est la seule société non-artistique à qui la municipalité veut bien octroyer le théâtre jusqu'en 1922. On ne compte pas le nombre de spectacles donnés au profit des associations caritatives et philanthropiques nées de la guerre. Tournée du théâtre des Variétés d'Angers au profit des soldats tuberculeux, séance cinématographique spéciale en l'honneur des "gueules cassées " ou encore concert d'une société musicale à l'attention des orphelins de guerre... le récent conflit reste le thème récurrent des premières années d'après guerre au théâtre.

\section{Flottements sur les planches et errements administratifs}

Avec l'arrêt des combats, le comité devrait se trouver dissous ${ }^{11}$. Pourtant, peu après mars 1919, c'est le comité permanent des fêtes qui reprend le flambeau, avec les mêmes activités et les mêmes objectifs. Les bénéfices sont intégralement reversés " au bureau de bienfaisance et aux œuvres philanthropiques, mutilés de guerre, pupilles de la Nation, etc. ${ }^{12}$ ». En 1920, la décision de se servir d'une partie des recettes comme subvention aux festivités du comité permanent des fêtes amène la municipalité à réagir, sur la demande du cinéma Palace qui y voit non plus une exploitation philanthropique mais commerciale. Le théâtre est mis en adjudication et le comité permanent des fêtes doit, en mars 1920, « suspendre les représentations cinématographiques afin de laisser entière liberté à la municipalité ${ }^{13}$ ". Ce qui n'était, à l'origine, qu'un palliatif culturel durant les hostilités a fait long feu.

L'intérêt d'adjuger le marché de la location du théâtre avec publicité et concurrence est simple : le théâtre est un équipement qui coûte cher et rapporte peu ${ }^{14}$; donner sa gestion à un entrepreneur privé sous forme de bail permettrait d'équilibrer cette ligne budgétaire. Comment réglementer ce système? Dans un premier temps, la municipalité se renseigne en inter-

11. Arch. mun. de Cholet. Délibérations du conseil municipal, 26 août 1919. Le propriétaire du cinéma Palace, qui y voit un concurrent avant tout, fait des remarques en ce sens.

12. Ibidem. C'est la justification du conseil municipal pour sa décision de maintenir le comité de fêtes comme exploitant de cinéma au théâtre municipal.

13. L'Intérêt Public, 7 mars 1920.

14. Arch. mun. de Cholet, 1L3, budgets primitifs. 
rogeant les théâtres d'Angers, Nantes, Le Mans, Rennes, Tours et Cognac ${ }^{15}$. Les plaintes répétées du propriétaire du cinéma Palace, à propos d'une concurrence illicite, conduisent la municipalité à être prudente sur le sujet, voire frileuse. Pour l'avoué de la ville, " [les pièces du dossier] démontrent bien que la location des théâtres ne se fait pas à l'adjudication ${ }^{16}$ ".

Un cahier des charges est tout de même établi et approuvé au conseil municipal. Il est adressé à plus de 96 professionnels du spectacle, entrepreneurs, journaux et magazines spécialisés pour créer la concurrence et s'assurer une hausse des enchères. Cet envoi donne lieu, " de la part d'adjudicataires éventuels, à des observations dont la plus importante est relative à la durée de la concession ${ }^{17}$ ". Des modifications sont apportées en ce sens puis, rendez-vous est donné à l'hôtel de ville, le vendredi 16 avril 1920. Un procès-verbal d'adjudication est préparé avec une très longue explication des procédures d'affermage. C'est un fiasco. Aucun adjudicataire ne se fait connaître et aucun compte rendu de l'adjudication n'est donc rédigé! Le temps passe, les démarches piétinent... Dans les mois qui suivent, la municipalité reçoit de nombreuses propositions d'entrepreneurs très peu versés dans le spectacle mais prêts à faire des affaires : le grossiste en boisson Montano des Sables-d'Olonne ou encore Lallier, le délégué du bureau national des Charbons. Résignée, la municipalité décide de supporter entièrement la gestion du théâtre.

L'année suivante, la ville de Cholet trouve une ultime occasion d'échapper à la gérance qui lui est imposée du théâtre et des tournées, ce dernier terme désignant ce que l'on appelle aujourd'hui une compagnie. Il est alors question de signer une convention avec les frères Grelu. Avec leur cabaret, les deux hommes vendaient déjà du loisir populaire avant la guerre. En 1918, ils ouvrent un cinéma stratégiquement placé entre la caserne du $77^{\mathrm{e}}$ Régiment d'Infanterie et la maison close de la rue du lait de beurre... Ils diversifient maintenant leurs activités avec un projet qui consiste à mettre en place de longues séries de concerts estivaux dans le jardin du Mail. En échange de la gratuité de l'endroit, " pendant la saison d'hiver du $1^{\mathrm{er}}$ octobre au 31 mars, un minimum de huit journées de représentations théâtrales ou séances artistiques sera donné dans l'édifice dit théâtre municipal ${ }^{18}$ ". Les concerts d'été sont un succès, sûrement très profitable, mais les frères Grelu ne donnent aucun spectacle au théâtre l'hiver suivant.

\section{Le règne de Camille Gaultier}

Les deux principaux maires qui gouvernent entre les deux guerres ne s'intéressent pas au théâtre. Un homme a donc les pleins pouvoirs pour

15. Arch. mun. de Cholet. Correspondance du maire, 24 janvier 1920.

16. Arch. mun. de Cholet. Dossiers préparatoires du conseil municipal, 24 février 1920 .

17. Arch. mun. de Cholet. Délibérations du conseil municipal, 3 avril 1920.

18. Arch. mun. de Cholet, 2R32, 23 juin 1921. 
gérer l'édifice et la programmation, c'est le secrétaire général Camille Gaultier. Cet employé, entré dans l'administration municipale en 1898, quitte son poste en 1941 : la politique de la ville à l'égard du théâtre reste donc constante. C'est un travail qui doit lui tenir à cour puisqu'il n'a pour cette charge " aucune rémunération spéciale ${ }^{19}$ ». Dans la plupart des correspondances à ce sujet, ce n'est pas au nom du maire qu'il répond, mais personnellement. Dans une lettre à un impresario, il explique, à propos de la remise accordée aux tournées, qu'« il n'y a pas lieu de s'inquiéter car c'est toujours moi qui suis chargé de l'accorder ${ }^{20}$ ». La preuve de son importance est également faite dans cette lettre d'un entrepreneur de Saint-Nazaire souhaitant louer le théâtre. Après de vaines négociations avec Camille Gaultier, il s'adresse directement au maire : "Sur les conseils de monsieur votre fils, qui m'avait dit, ce que le secrétaire fera, mon père le fera, je suis donc allé voir ce dernier auprès de qui j'ai rencontré une hostilité non déguisée; [...] j'ai donc vu à son attitude qu'une candidature à la direction du théâtre avait l'heur de lui déplaire; d'autre part, connaissant les sentiments de votre secrétaire sur cette question, beaucoup de Choletais m'avaient déconseillé une démarche auprès de lui comme n'ayant aucune chance d'aboutir ${ }^{21}$." La lettre est sans réponse. L'influence du secrétaire général est donc indéniable et la gestion du théâtre est sa chasse gardée.

" Le théâtre municipal [...] reste le point d'ancrage de la vie culturelle de bien des villes de province. Et très souvent, c'est la question de sa gestion qui est à l'origine des premiers débats de fond sur la légitimité d'un service public municipal dans ces domaines ${ }^{22}$. " À Cholet, les premières discussions interviennent en effet après l'échec de la mise en adjudication. Un revers finalement relatif et, peut-être, souhaité par Camille Gaultier : " Monsieur Mollé, en essayant d'obtenir de lui quelques renseignements sur ce qui avait été proposé en mars 1920 lors de la mise en adjudication s'est heurté à un mauvais vouloir ${ }^{23}$. " Toutes les demandes postérieures de location du théâtre sont rejetées. C'est un fait acquis, le théâtre est géré par la ville, ce qui lui permet au final d'en disposer pour d'autres usages.

\section{La gestion municipale du théâtre}

La gestion du théâtre implique toute une organisation, à commencer par celle du personnel, c'est-à-dire six ouvreuses, cinq contrôleurs, trois policiers, trois pompiers et un balayeur ${ }^{24}$. Tous doivent être rémunérés à chaque représentation car il ne s'agit pas d'activités à temps complet. Même les pompiers et les policiers perçoivent un salaire pour ces perma-

19. Arch. mun. de Cholet. Correspondance du maire, 23 mars 1923.

20. Arch. mun. de Cholet, 2R36.

21. Arch. mun. de Cholet, D7, liasse 130, 22 mars 1921. Lettre de Maurice Benoist.

22. POIRRIER, Philippe, L'État et la culture en France au XXe siècle, Paris, Le livre de poche, coll. "références ", 2000, p. 28.

23. Arch. mun. de Cholet, D7, liasse 130, 22 mars 1921.

24. Arch. mun. de Cholet, 2R32, saison 1918-1919. 
nences effectuées en dehors de leurs heures de travail réglementaires. " $\mathrm{La}$ machinerie du théâtre est assurée par un chef qui s'occupe du recrutement de ses aides et doit assurer la fourniture des accessoires [...]. En outre, le chef doit fournir la main-d'œuvre pour aller chercher et retourner en ville le mobilier nécessaire aux spectacles. Pour l'ensemble des services, il reçoit une indemnité forfaitaire [...]. Deux électriciens au service de la ville reçoivent également une indemnité $[\ldots]^{25}$." Ce sont donc au moins 24 personnes qui sont mobilisées à chaque représentation théâtrale. Il est par ailleurs fréquent que des amateurs viennent bénévolement en aide aux professionnels. Exemple avec l'imprésario Le Quilliec, qui vient jouer Les linottes le 20 avril 1927 au théâtre municipal : il sollicite un orchestre local de quatre instruments pour enrichir la partie musicale, " sinon le chef jouera seul au piano ${ }^{26}$ ".

Autant que faire se peut, la municipalité essaie de reporter les charges sur les tournées, d'où l'établissement d'un bordereau de frais, envoyé à toutes les troupes désirant passer à Cholet. En 1924, il est composé comme suit : 142 francs de frais de salle et de personnel, 20 francs de camionnage, 60 à 80 francs d'affichage, 6 \% de la recette brute pour la taxe d'État, le droit des pauvres et les droits d'auteur ${ }^{27}$. Voilà pour la théorie.

En pratique, ces frais sont peu souvent assumés par les tournées : "L'administration municipale en vue de favoriser, dans la mesure du possible, les directeurs de tournées, leur consent une réduction sur les frais de salles et d'éclairage, évalués à 160 francs par séance ${ }^{28}$. " Il y a ensuite les remises qui, dans l'absolu, sont octroyées le jour du spectacle selon le barème suivant : recette nette égale ou supérieure à 1801 francs, sans remise; recette nette de 1701 à 1800 francs, remise de 25 francs; recette nette de 1601 à 1700 francs, remise de 50 francs; recette nette de 1501 à 1600 francs, remise de 75 francs; recette nette égale ou inférieure à 1500 francs, remise totale ${ }^{29}$. Aucun des six spectacles présentés par le cycle Charles Baret en 1923-1924 ne dépasse les 1500 francs de recettes nettes ${ }^{30}$. La Société des auteurs et compositeurs dramatiques se plaint régulièrement de n'être pas payée et les négociations sont longues avant la signature d'un contrat.

La gestion du matériel est assurée par le chef machiniste, mais la création des décors n'est pas dans ses attributions. La plupart des tournées viennent cependant sans décors, comptant sur ceux multifonctions mis à disposition. En 1923, le théâtre dispose d'un salon moderne, d'un salon bourgeois et de deux paysages dont un rustique.

25. Arch. mun. de Cholet. Correspondance du maire, 11 juin 1930. Lettre du maire à son homologue saumurois.

26. Arch. mun. de Cholet, D7, liasse 135, 16 avril 1927.

27. Arch. mun. de Cholet. Dossiers préparatoires du conseil municipal, 8 mars 1924.

28. Arch. mun. de Cholet. Correspondance du maire, 20 mai 1936.

29. Arch. mun. de Cholet. Délibérations du conseil municipal, 10 décembre 1930.

30. Arch. mun. de Cholet. Dossiers préparatoires du conseil municipal, 8 mars 1924. Les recettes nettes des spectacles du cycle Baret oscillent entre 518 et 1448 francs. 
Il faut ajouter à la logistique du théâtre la multitude de détails essentiels comme, par exemple, la billetterie ${ }^{31}$ ou encore la gestion des "servitudes et places de faveur ". Il s'agit du placement de toutes les personnes nécessaires au bon déroulement de la séance et des personnalités locales. Une loge est réservée au sous-préfet, une au maire et une aux adjoints. Les conseillers municipaux ont deux fauteuils, le secrétaire général un, de même que l'officier des pompiers. Le directeur du Palais des marchands (grand magasin choletais), qui fournit gratuitement les meubles et les accessoires, a deux fauteuils; l'architecte voyer, deux stalles; le commissaire de police, deux places de seconde. Certaines places échappent au contrôle de l'administration municipale : six fauteuils pour la société des auteurs dramatiques et son représentant choletais (Albert Birot), trois fauteuils pour la société des auteurs lyriques et sept fauteuils pour la presse locale, régionale et l'agence d'affichage. En tout, 38 places sur 498 (avec les restrictions de l'architecte voyer pour des raisons de sécurité) sont réservées gratuitement, ce qui n'arrange rien pour le problème de rentabilité du théâtre. Camille Gaultier résout cette question soulevée par l'administration Baret en consentant à ce qu'il " soit placé un rang de chaises, 20 environ, dans la partie réservée à l'orchestre ainsi que dans les couloirs de dégagement ${ }^{32}$ ", rendant, soit dit en passant, caduques les précautions de l'architecte voyer sur la sécurité des dits couloirs.

La municipalité doit également se pencher sur les finances. Le théâtre n'étant pas adjugé, le maire demande régulièrement au préfet " une remise des impôts fonciers afférents au théâtre municipal ${ }^{33}$ ". En vain. Une solution est trouvée en 1925 : la publicité. Trois compagnies assaillent la mairie depuis un moment et la municipalité signe un contrat avec la Publicité de l'Ouest et du Centre ${ }^{34}$. Pour une période de cinq ans, cette société s'occupe de la mise en place d'un rideau de réclame et paye 1200 francs par an à la ville de Cholet. Le rideau reste propriété de la ville, mais celle-ci doit évidemment le manœuvrer pendant les représentations et les réunions. Il est prévu d'affecter la somme obtenue à l'entretien du mobilier du théâtre. En 1931, le contrat n'est pas renouvelé car "nos représentants sont allés, hier, visiter les commerçants choletais. Ils ont reçu de nombreuses plaintes relatives à la mauvaise manœuvre du rideau, celui-ci n'étant pas baissé à chaque représentation ${ }^{35}$ ". Le maire réplique tout de même qu'il n'a été saisi " d'aucune plainte relative à la manœuvre dudit rideau ". La municipalité se voit privée d'une intéressante source de financement, d'autant plus utile que la période est délicate pour le théâtre et que les concessions financières doivent se multiplier pour faire venir les tournées.

31. Arch. mun. de Cholet, 2R32. Choix de la société Numerus pour le service de billetterie.

32. Arch. mun. de Cholet, 2R39. 9 septembre 1925.

33. Arch. mun. de Cholet. Correspondance du maire, 11 juillet 1922, 6 janvier 1938.

34. Arch. mun. de Cholet, 2R32, 28 janvier 1926.

35. Arch. mun. de Cholet, 2R32, 14 mars 1931. 
Le théâtre est en permanence source de dépenses. Régulièrement, le mauvais état du mobilier fait l'objet de remarques au conseil municipal. À partir de 1925, une nouvelle ligne budgétaire est donc inscrite dans les comptes de la ville : " achat et entretien du mobilier du théâtre ", 1000 francs tout d'abord puis, dès 1926, 2000 francs. Cette somme est invariablement annoncée dans les budgets primitifs jusqu'en 1940. Les comptes administratifs révèlent très peu de dépassements (sauf en 1927 pour la réfection de tous les fauteuils). La question de l'électrification du théâtre apparaît en 1927, en même temps qu'une " sous-commission théâtre " au conseil municipal. En août, une somme de 33000 francs est votée pour le projet d'électrification. Sa réalisation coûte un peu plus de 40000 francs.

\section{La valse des tournées : Camille Gaultier face au choix}

Les professionnels du théâtre ne tardent pas à retrouver le chemin de la province après la guerre et, notamment, celui qui mène à Cholet. Hernani de Victor Hugo est la première représentation donnée par des professionnels, le jeudi 5 juin 1919. Quinze autres spectacles investissent la scène du théâtre municipal entre juin et décembre 1919. On peut parler d'activité intense puisqu'un peu plus de deux troupes par mois jouent devant les Choletais. Les saisons suivantes ne sont pas toujours aussi bien servies. Ce dynamisme du théâtre professionnel s'explique par l'engouement qu'il suscite chez un public qui en a été privé pendant cinq ans, mais aussi parce qu'aucune contrainte administrative ne pèse sur l'utilisation du lieu par des tournées. Jusqu'en janvier 1920, il n'est pas question d'adjudication. Cette affaire a sûrement un effet positif à moyen terme car, le cahier des charges étant envoyé dans les centres névralgiques de l'activité théâtrale professionnelle, le théâtre de Cholet se fait connaître. Une fois la gestion municipale assumée, Camille Gaultier voit affluer de nombreuses demandes, parfois de la part de directeurs de théâtre et, surtout, de la part des impresarios.

Les courriers de directeurs de théâtre sont relativement rares. Paul Douai, en 1930, tend une main généreuse vers Cholet. Nouveau directeur du théâtre d'Angers pour la saison 1930-1931, il annonce vouloir " donner des spectacles, qui, comme valeur artistique, n'auront rien à envier à ceux qui ont pu être donnés jusqu'à ce jour ${ }^{36}$ ". Il se targue de son expérience passée, dans quatre villes du nord de la France. Désireux de diffuser l'art théâtral ou soucieux de rentabiliser les voyages des tournées, il propose " d'excursionner " sur Cholet. Un peu plus tard, il précise réserver le mardi comme jour de passage et indique qu'il est prêt à assurer une régularité d'un mardi par mois. Ces propositions sont tout à fait occasionnelles.

Dans la grande majorité des cas, c'est la tournée elle-même qui démarche la municipalité. Elle porte généralement le nom de l'imprésario ou du théâtre auquel elle est rattachée. Ces compagnies sont légion dans l'entre-

36. Arch. mun. de Cholet, 2R32, 16 janvier 1930. 
deux-guerres. Elles envoient des publicités parfois très simples et sobres, souvent riches et clinquantes, démontrant toute la diversité de cet univers ${ }^{37}$. Le nombre des demandes suit, évidemment, la vitalité du milieu théâtral. Le graphique ci-dessous met en évidence l'effervescence de ce secteur jusqu'en 1930 où les demandes de passage à Cholet atteignent le nombre record de 57 . L'année suivante reste dans la moyenne de la décennie avec 46 demandes. En 1932, on dégringole à 29 demandes. Le monde du théâtre est alors secoué par une violente crise dont nous reparlerons.

Nombre de sollicitations par des impresarios

(Source : Arch. mun. de Cholet, 2R35, 2R36)

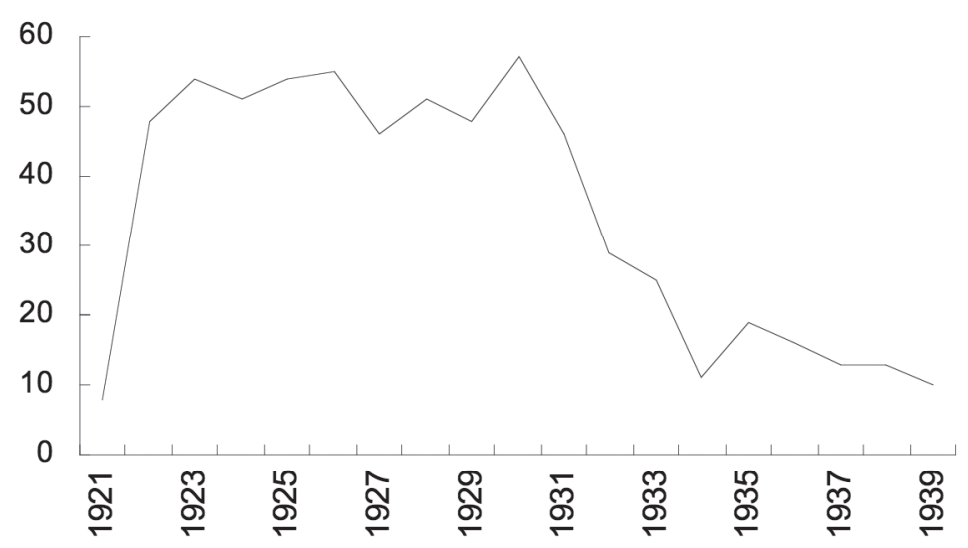

Pendant la période fastueuse de 1922 à 1931, Camille Gaultier voit arriver une cinquantaine de propositions tous les ans. Quelles sont ces tournées? Certaines reviennent régulièrement et méritent d'être citées : Georges Zeller, René Forval, Fernand Volric, Jeanne d'Orsay, Fernand Doryval, Marcel Duvernot, Clevers et autres Jean Valmy sont autant d'imprésarios qui font les grandes heures du théâtre municipal de Cholet. Dans les années 1930, beaucoup disparaissent et de nouveaux noms font leur apparition tels Catriens, Mayol, Jan Bertran, etc.

Les tournées proposent en grande majorité des comédies de boulevard et des drames à la mode, quelques revues et opérettes, parfois des classiques ${ }^{38}$ et plus rarement des spectacles de magie ou de spiritisme. L'année 1922 est représentative des propositions faites. Le Boulevard est imposé

37. Arch. mun. de Cholet, 2 R35 \& 2R36. Avec les échanges répétés de courrier, il y a parfois plus de 200 lettres pour une seule année. Chaque troupe possède sa personnalité; notre ambition est de montrer les traits marquants des relations avec cette corporation.

38. Par classique, il faut entendre les œuvres du répertoire français non contemporaines à l'époque. 
au programmateur dans $65 \%$ des cas. "Cet art du pur divertissement qui joue sur une intrigue bien ficelée, des thèmes aguicheurs et sécurisants, et des mots d'auteurs, est le plus souvent méprisé par les artistes et les intellectuels ${ }^{39}$."

Genres proposés par les impresarios pour l'année 1922

(Source : Arch. mun. de Cholet, 2R35, 2R36)

\begin{tabular}{|l|l|l|}
\hline Boulevard & 31 & soit $65 \%$ \\
\hline Opérette & 6 & soit $13 \%$ \\
\hline Revue & 4 & soit 8\% \\
\hline Répertoire classique & 4 & soit 8\% \\
\hline Magie et spiritisme & 3 & soit 6\% \\
\hline
\end{tabular}

La proportion de ce que les tournées offrent n'est pas forcément significative de ce que Camille Gaultier choisit pour son public. Comment opèret-il? Première solution pour le secrétaire général, se renseigner auprès d'un homologue dans une ville ayant déjà accueilli la tournée concernée. C'est pourquoi les impresarios indiquent toujours de multiples références. Il arrive également que l'administration municipale réclame le texte de la pièce avant d'accepter une tournée. Lorsqu'il s'agit d'une œuvre connue, l'imprésario s'empresse de mentionner que « la mise en scène impressionnante évoquera fidèlement dans l'esprit de votre public les différentes phases du roman qui aura été lu par tous ${ }^{40}$ ". Il n'y a aucune hésitation lorsqu'il s'agit d'une pièce " au service d'une propagande utile au progrès des mœurs ${ }^{41}$ ", comme c'est le cas par exemple pour Mortel baiser, patronné par la Ligue nationale française contre le péril vénérien. Autre genre accepté sans difficulté, les conférences documentaires, proposées souvent par Paul Boquel, dont les thèmes tournent régulièrement autour des colonies avec des récits d'explorateurs : "L'intérêt est national [avec] une profonde volonté pédagogique ${ }^{42}$."

Sur une cinquantaine de demandes dans les années 1920, un peu moins d'une quarantaine reçoit un accord de principe et une quinzaine est rejetée directement. Dans ce cas, le secrétaire général envoie une formule aux allures de mensonge : "Le théâtre est retenu pour toute la saison ${ }^{43}$."

En matière de publicité, les impresarios ne sont pas en reste. Ils développent d'astucieuses techniques de vente, racoleurs juste ce qu'il faut,

39. EvRARD, Franck, Le Théâtre français du XX siècle, Ellipses, 1995, Paris, page 110.

40. Arch. mun. de Cholet, 2R35, 1926. Lettre d'un impresario à propos de la pièce Satanas de Gabriel Bernard.

41. Arch. mun. de Cholet, 2R35. Lettre d'un professeur en médecine accompagnant la publicité de "Mortel baiser".

42. Arch. mun. de Cholet, 2R35. Lettre de Paul Boquel.

43. Arch. mun. de Cholet, 2R36. Réponse, par exemple, à l'imprésario de La fille du bédouin. 
démonstratifs au possible, dans toutes les gammes susceptibles d'attirer l'attention du destinataire. La comédie commence parfois dans le courrier des tournées. Pour la pièce Les dégourdis de la 11e, l'imprésario garantit " du bon rire, bien sain et bien français [qui] amuse follement tous les publics et peut être vu par tous ${ }^{44}$ ". Polin, qui propose La folle escapade, assure lui aussi : "Papas, mamans, vous pouvez y conduire vos enfants. " Quant à la pièce Kiki fait cocu Coco, dont on peut facilement imaginer la finesse et la subtilité, la tournée ne promet rien de moins que " 300 éclats de rire, trois actes ultra-comiques, trois heures de folle gaîté [...] un gros succès du rire ", avant de préciser, " pièce amusante qui ne choque personne, jouée par des vedettes de Paris ". Il y a là tous les poncifs de l'argumentation des impresarios : faire français et patriote, garantir du rire à tour de bras et ne surtout pas choquer!

La province est réputée moins ouverte aux innovations. Les tournées qui osent s'aventurer avec un texte plus élaboré, et non pas du rire facile, prennent bien la peine de préciser qu'il n'y a pas de danger lorsque ce n'est pas du théâtre classique. C'est le cas de l'adaptation de La garçonne; la brochure de l'imprésario Jules Mati est agrémentée de 17 avis. Journaux, élus municipaux et autres autorités clament que la pièce n'a rien à voir avec le roman et qu'elle peut être vue par tous ${ }^{45}$.

Autre domaine, autres clichés servis à tous les coups : les spectacles mystérieux et surnaturels. L'exemple de la tournée Carrington est assez typique de l'esprit de mystification des impresarios avant même la représentation! Son siège social est à Louviers dans l'Eure, mais " l'homme qui joue avec le surnaturel ", bien français, est assurément plus crédible dans sa version britannique. Ce genre de spectacle se targue de ses circuits à travers l'Europe, voire le monde entier, comme « le psychographe spirite Donato et Yvonne Chaumont son sujet " qui, en permanence, reviennent tout juste d'une grande tournée américaine... On peut aujourd'hui poser un regard amusé sur ces brochures, mais elles sont à l'époque la quasi unique source de renseignements pour le secrétaire général chargé de la programmation.

Sur le principe, c'est le plaisir des administrés qui semble être déterminant dans les choix, au point que la fameuse remise, systématiquement réclamée par les tournées lors de leur passage, n'est accordée qu'en "se basant sur la satisfaction du public ${ }^{46}$ ». Camille Gaultier refuse fréquemment des pièces en se fiant à leur insuccès passé. "Vous n'avez d'ailleurs pas été autorisé à jouer La Tosca, votre passage avec Mignon ayant fait l'objet de vives critiques de la part du public ${ }^{47}$ ". Le retour d'une tournée d'une année sur l'autre, parfois dans la même saison si celle-ci est assez importante pour supporter plusieurs spectacles en même temps (c'est un cas assez fréquent), est signe de satisfaction. Par de simples échanges courtois, des

44. Arch. mun. de Cholet, 2R35, 1923.

45. Arch. mun. de Cholet, 2R36, 10 avril 1928.

46. Arch. mun. de Cholet, 2R36.

47. Arch. mun. de Cholet, 2R35, 18 décembre 1922. Lettre à l'imprésario Jean Dubos. 
tics de langage qui trahissent l'habitude de la relation avec tel ou tel, on peut induire que les rapports sont au beau fixe avec certaines tournées qui ont leurs marques à Cholet. Camille Gaultier ne le cache pas : " Nous ne pouvons vous réserver cette date [...] ayant pris l'habitude d'accueillir les tournées Valmy dans ces jours de décembre ${ }^{48}$."

\section{L'imbroglio des impresarios}

Ce n'est pas toujours la courtoisie qui régit les relations épistolaires entre Camille Gaultier et ses correspondants. Les uns n'acceptent pas de concurrence trop rapprochée, les autres veulent réduire les frais de salle et certains tiennent absolument à vendre leur pièce, jusqu'à la fâcherie. Les rapports sont plus épicés à partir de 1932, date qui correspond à la chute du nombre de demandes. En août 1933, le secrétaire général refuse par exemple Les sorcières de l'occident par les tournées Jack Dorival parce que, selon lui, " ce genre de spectacle n'a pas de succès à Cholet ". D'où la réponse du directeur de la tournée quelques jours plus tard, adressée cette fois au maire : "Au titre de Français et de mutilé pensionné de guerre cinq blessures, je serais désireux de savoir les autres dates que vous avez de disponibles pour faire mes représentations et présenter à monsieur le secrétaire général mes excuses et lui donner une petite leçon, il est vrai que ce monsieur fréquente peut-être d'autres établissements de votre bonne ville ou est-il le secrétaire d'une vague société de spectacles ou encore est-il représentant d'une maison de film étrangère. Il est vrai que les étrangers sont maîtres en notre pays et la bonne ville de Cholet a le droit de présenter à ses administrés des pièces de quatrième catégorie ${ }^{49}$. " Panique d'une société de spectacle sur le déclin ou délire d'imprésario sur fond d'Action Française? Quoi qu'il en soit, les rapports sont parfois musclés. Autre exemple, en 1933 toujours, un esclandre avec Maurice Le Drazal, dû cette fois aux cafouillages de la municipalité. Camille Gaultier accepte une date puis revient sur sa décision " après nouvelle étude de la question ". Le Drazal répond : "Je ne puis que me contenter de juger la municipalité de Cholet telle qu'elle me jugerait si j'avais agi envers elle avec la même désinvolture incorrecte. D'ailleurs, je me propose de soumettre le cas à M. Audier, co-directeur des tournées Baret qui est président de la chambre syndicale des directeurs de tournées de France ${ }^{50}$. "Les échanges de courrier s'enflamment sur des propos sans grand intérêt jusqu'à ce que Camille Gaultier clôture le dialogue en notant sur la dernière lettre : « Ne pas répondre. " Il est nécessaire d'imaginer combien il doit être difficile pour le secrétaire de mairie d'une ville de 20000 habitants de gérer une entreprise aussi lourde, avec des interlocuteurs souvent capricieux et, pour la plupart, totalement inconnus.

48. Arch. mun. de Cholet, 2 R35.

49. Arch. mun. de Cholet, 2R36, 23 août 1933.

50. Arch. mun. de Cholet, 2R36, novembre 1933. 
L'éloignement ou l'ignorance mène parfois à quelques erreurs de jugement. Les tournées théâtrales sont-elles vraiment composées " d'illustres auteurs ", de " fameux comédiens " ou encore de " stars parisiennes ${ }^{51}$ " ? Un article de Maurice Prax du Petit Parisien, de retour d'un voyage en province, prévient avec ironie : " Les plus grands succès de l'époque ont des titres qu'il m'est, hélas! impossible de reproduire ici. Ils sont à la fois idiotement sales et salement idiots. [...] Quant aux illustres auteurs contemporains, ma foi, ils ont de drôles de noms et des noms que nous n'avons jamais entendus prononcer à Paris. Les prestigieuses affiches nous garantissent cependant qu'ils sont célèbres. Devons-nous en douter? J'ai vu hier un échantillon de leurs productions qui tenait du prodige. Il n'était pas possible de faire plus bête, plus lourd, plus ennuyeux, plus crapuleux. C'était du délire dans l'ineptie... [...] Il y a ainsi quelques misérables tournées, sans références ni traditions, qui promènent à travers la France, des spectacles imbéciles et indécents ${ }^{52}$. " Et le journaliste de continuer en fustigeant ces " anonymes tâcherons ", ces "minables mercantis ". Cet article, sûrement découpé par Camille Gaultier, a été placé dans les dossiers de candidature des tournées. Il montre encore à quel point il est difficile pour un provincial, quand bien même serait-il averti, de démêler les charlatans des vrais artistes.

L'image de la capitale est une réalité bien souvent déformée, car amplifiée par quelques bourgeois cabotins (René Creusé est une figure choletaise exemplaire) ou bien magnifiée par des critiques locaux qui n'ont qu'une connaissance lointaine des authentiques productions parisiennes. La capitale demeure la référence culturelle, quand bien même les aspirations de la province sont totalement en décalage. Avec un peu de condescendance, Charles Baret met en garde contre les supercheries : "Nous parlons de véritables vedettes et non pas des noms que certains impresarios impriment en grosses lettres dans le but évident de tromper la province. [Ces impresarios] cherchent à battre monnaie en province parce que la masse du public y est bien moins renseignée qu'à Paris ${ }^{53}$. " La maison Baret fonde son commerce sur sa renommée et tient à montrer sa compréhension des différences de goût entre la province et Paris : "Une pièce obtient à Paris un succès vif, persistant, et la province la trouve ridicule, franchement mauvaise. " Jamais le secrétaire général de la mairie ne reçoit de publicité d'un impresario pour faire venir une pièce d'André Gide ou de Jean Cocteau, jamais des acteurs tels Charles Dullin ou Louis Jouvet ne s'exportent dans son courrier... Cholet n'est pas en marge du goût populaire de l'époque pour un théâtre "beaucoup plus conventionnel [qui] flatte les goûts d'un public qui aime le rire facile et la sentimentalité à fleur de peau ${ }^{54}$ \%. Le reste, même dans la capitale, n'est que le fait d'une élite restreinte.

51. Arch. mun. de Cholet, 2R35 et 2R36. Brochures et publicités.

52. Arch. mun. de Cholet, 2R39, 10 mars 1925. Coupure de journal tirée du Petit Parisien.

53. Arch. mun. de Cholet, 2R36. Brochure de présentation du cycle Baret pour la saison 1926-1927.

54. VersinI, Georges, Le théâtre français depuis 1900, Paris, PUF, coll. "Que sais-je? ", 1991, p. 11. 


\section{Programmation et fréquentation au théâtre municipal}

Seules $30 \%$ des demandes, entre 1920 et 1930, débouchent sur une représentation au théâtre municipal. Il n'est pas dans les habitudes de programmer beaucoup de pièces au printemps et à l'automne, et $a$ fortiori en été. L'essentiel des tournées passe entre novembre et février. Celles-ci jouent généralement le mardi ou le jeudi, rarement pendant les congés de fin de semaine. Les réjouissances sont généralement longues et les soirées de gala n'hésitent pas à faire s'enchaîner deux pièces. Les revues ont également pour habitude d'être très étendues dans le temps. Camille Gaultier ne rate jamais une occasion de programmer un classique, puisque tous ceux qui lui sont proposés sont sélectionnés. Une sorte de tradition exige une ou deux revues pour les fêtes de fin d'année. Les spectacles de magie et de spiritisme, s'ils viennent parfois à Cholet, sont sous-représentés par rapport au nombre de sollicitations des impresarios. C'est le théâtre de Boulevard et les drames à la mode qui occupent à égalité la scène. "Avec ou sans intentions sociales et satiriques, [les comédies de situation et d'intrigues du Boulevard] répondent à l'attente du public à la recherche d'un imprévu... prévisible ${ }^{55}$. "Et l'historien de citer Les vignes du seigneur de Flers ou encore La dame de chez Maxim de Feydau, deux grands succès du théâtre municipal de Cholet. Faut-il blâmer Camille Gaultier de faire une programmation trop "facile", alors qu'il est dépendant de ce que les impresarios lui proposent et des attentes du public? « Prenant appui sur des thèmes galvaudés comme la frivolité de l'univers bourgeois, les jeux de l'amour et du hasard, les entorses à la morale traditionnelle, ce théâtre [...] continue à séduire un public peu motivé par le théâtre à thèse ${ }^{56}$. " Le secrétaire général offre tout de même du choix. Les Choletais peuvent s'amuser avec Un beau-père pas commode, Odette dépêche-toi, Quatre femmes pour un caporal ou encore Tu verras ma tante..., mais ils ont également accès à Hernani, Le Cid, Polyeucte ou encore les Noces de Figaro.

Une fois le spectacle programmé, la fréquentation le soir même est diffcile à mesurer. Seuls les courriers des tournées, de plus en plus alarmistes au fil de la période, peuvent donner une idée de la baisse régulière de la fréquentation du théâtre. Et encore... sans faire salle comble à tous les coups, on peut s'interroger sur ces manques à gagner systématiques lors des passages à Cholet. Les tournées Baret répondent qu'" en raison des frais importants qu'elles ont à supporter et du petit nombre de places que contient le théâtre ${ }^{57}$ ", il est difficile de rentrer dans ses frais. On croise donc régulièrement le paradoxe suivant : "Vous savez que les recettes faites au théâtre de Cholet ne sont pas très élevées, [...] le gros succès d'hier soir en est une nouvelle fois la preuve ${ }^{58}$. " Le théâtre municipal n'est pas rentable commercialement. La notion de fréquentation est donc sujette à

55. EVRARD, Franck, Le théâtre français..., op. cit., p. 13.

56. Ibidem, p. 27.

57. Arch. mun. de Cholet. Délibérations du conseil municipal, 9 décembre 1933.

58. Arch. mun. de Cholet, 2R39, 30 septembre 1923. 
caution car même un succès de ce point de vue peut être considéré comme un four financier par l'imprésario.

\section{Charles Baret : le Napoléon des tournées et Cholet}

Il est nécessaire de distinguer la compagnie Charles Baret, parce que son fonctionnement est particulier et parce qu'elle a su créer un lien fort avec la ville de Cholet. Plus que des tournées, le célèbre impresario a l'idée de proposer un cycle. "Le cycle c'est la tournée achevée ", exulte Charles Baret dans une luxueuse brochure présentée pour la saison 1926-1927. Il s'agit de proposer à la ville, non pas une tournée occasionnelle, mais une série de pièces programmées à l'avance sur toute une année, une aubaine pour Camille Gaultier qui garantit ainsi un minimum de six, huit, voire dix pièces au théâtre municipal, et une habile opération commerciale pour Charles Baret qui s'assure la fidélité des villes et des cachets un an à l'avance. Seule une entreprise assez solide peut se permettre une telle organisation, mais la réputation des tournées Baret est bien ancrée en province comme à Paris. "Charles Baret, directeur de la plus vaste entreprise de tournées artistiques que nous possédions en France, est l'honneur d'une profession où la probité est exposée à toutes sortes de périls ${ }^{59}$ ", écrit l'académicien Robert de Flers, le 8 août 1923. À la demande de son administrateur, la compagnie obtient la priorité dans le choix des dates à Cholet.

Les abonnements sont une autre astuce de Charles Baret. Fort de son système de cycle, il peut présenter dès le début de la saison, le programme de l'ensemble de l'année... et demander aux amateurs de théâtre de régler un forfait pour toutes ces représentations, moyennant une réduction intéressante. "Le système des abonnements n'ayant jamais été pratiqué à Cholet et connaissant le tempérament des habitués du théâtre, je vous souhaite une bonne souscription, sans trop oser y compter. Rien cependant, ne sera négligé ${ }^{60}$. " Malgré les réserves de Camille Gaultier, les abonnés sont 167 en 1926, ce qui satisfait pleinement l'administration Baret. Celle-ci adresse ses " remerciements pour ce brillant résultat " et peut ainsi faire tourner l'entreprise avec un apport de fonds conséquent à chaque début de saison.

Dernière originalité du fonctionnement de ces tournées, l'installation de correspondants dans chaque ville prévue dans le circuit du cycle. À la fin de 1941, lors du départ à la retraite de Camille Gaultier, on apprend que celui-ci était " représentant de la firme Baret ${ }^{61}$ " depuis le début, un élément qui peut expliquer le traitement de faveur dont le cycle est l'objet.

"Évidemment le cycle Baret engraisserait mieux sa caisse en exhibant certaines œuvres grivoises qui attirent toujours en foule un certain public $^{62}$ ", note la publicité des établissements Baret. Si la grande majorité

59. Arch. mun. de Cholet, 2R36, saison 1926-1927.

60. Arch. mun. de Cholet, 2R39, 3 septembre 1924.

61. Arch. mun. de Cholet, 2R39, fin janvier 1942.

62. Arch. mun. de Cholet, 2R36, saison 1926-1927. 
des pièces proposées est récente, elle n'entre pas dans le cadre des comédies de Boulevard traditionnellement proposées. "Si l'on avait demandé à un critique de 1910 de désigner les meilleurs auteurs dramatiques de son temps, il aurait sans doute cité Rostand, Hervieu, de Curel, Lavedan, Porto-Riche, Donnay, Brieux, Mirbeau, Bataille ${ }^{63}$. "Sur ces huit auteurs, les cycles Baret de 1924 à 1928 en proposent six (Hervieu, de Curel, Lavedan, Porto-Riche, Donnay et Bataille) et, souvent, plusieurs de leurs œuvres. À ces auteurs il faut ajouter Henry Bernstein, Tristan Bernard ou encore Paul Géraldy qui semblent avoir les faveurs de la programmation de Charles Baret. Celle-ci tranche sans conteste avec les propositions de la plupart des tournées, qu'il est possible d'étudier à partir des documents reçus à Cholet. Le cycle Baret, c'est aussi des pièces classiques jouées en intégral. Il n'y a pas une seule saison sans au moins une pièce de Molière ou de Corneille. Pour remplir les salles à ces occasions, l'administration Baret met en place " une publicité spéciale auprès des directeurs d'établissements scolaires $^{64}$ ", évoquant des réductions pour la jeunesse et les enseignants.

" Grâce au cycle, des théâtres, qui risquaient d'être désaffectés, connaissent plus que jamais la joie des beaux soirs. Pour certaines villes de 20000 à 30000 habitants, le cycle Baret est devenu un véritable bienfait. Il a créé de nouveaux aspects de la vie provinciale ${ }^{65}$. " Cet argument publicitaire n'est pas loin de la vérité. Les cycles ont indéniablement apporté un second souffle au théâtre de Cholet. La relation des tournées Baret avec la ville commence toutefois par un sacrifice de la part de la municipalité. " Nous n'osons pas encore inclure Cholet dans nos itinéraires de notre cycle de comédies, parce que les recettes réalisées dans votre ville [...] n'ont pas été brillantes. Il faudrait que comme dans nombre d'autres cités, votre municipalité consentît à nous aider, soit en garantissant un minimum, comme à Arles entre autres, soit en nous accordant une subvention en espèces pour chacun de nos passages ${ }^{66}$. " Une remise des frais de location est consentie ainsi qu'une prise en charge de plus de $50 \%$ de l'éclairage, preuve que les élus tiennent absolument à voir le cycle Baret faire halte à Cholet. La même année, lorsque la compagnie envoie une nouvelle demande similaire, celle-ci est tout de même rejetée pour des raisons budgétaires. Pourtant, après cet octroi unique de subvention en nature, le secrétaire général écrit à Charles Baret : "Comme vous avez pu vous en rendre compte, le public choletais a suivi régulièrement les spectacles de vos tournées et j'insiste à nouveau auprès de vous pour que notre ville soit comprise dans l'itinéraire de vos tournées ${ }^{67}$. " Est-ce le cycle Charles Baret qui courtise Cholet ou l'inverse? Après tout, le premier est une entreprise commerciale qui a besoin de la seconde pour vivre.

63. VERSINI, Georges, Le théâtre français..., op. cit., p. 7.

64. Arch. mun. de Cholet, 2R39, 9 novembre 1925.

65. Arch. mun. de Cholet, 2R36, saison 1926-1927.

66. Arch. mun. de Cholet, 2R39, 28 février 1924.

67. Arch. mun. de Cholet, 2R39, 25 avril 1925. 
Charles Baret n'échappe pas à la crise qui secoue le monde du théâtre à partir des années 1930 : augmentation des taxes, du transport des bagages, des chemins de fer, des cachets des artistes... les prix augmentent à trois reprises entre 1928 et 1930 . Un nouvel administrateur est mis en place à la tête des tournées Baret au début de l'année 1931, un signe d'instabilité à une époque où toutes les entreprises de ce genre montrent des faiblesses. Peu de temps après, les effets retenus de la crise éclatent enfin : "Malgré notre vif désir de maintenir Cholet dans nos itinéraires la saison prochaine, nous nous heurtons à une impossibilité absolue en raison des déficits importants que nous avons enregistrés cette année à chacun de nos passages et qui varient entre 1200 et 1500 francs par représentation. Comme nous ne pouvons pas demander à la municipalité de nous assurer contre le retour d'un pareil désastre, nous nous voyons dans l'obligation de répondre négativement à votre aimable proposition ${ }^{68}$. " Entre 1931 et 1933, Charles Baret déserte le théâtre municipal, jusqu'à la conclusion d'un nouvel accord qui exonère les tournées Baret des frais de bordereau (tous les frais inhérents à la location de la salle : chauffage, éclairage, personnel, droit d'auteur, affichage, etc.) soit une dépense de 346 francs par spectacle pour la municipalité. Les élus acceptent cette mesure de faveur " considérant que les tournées Baret, dont la réputation est indiscutable, sont les seules à donner dans notre ville, une série de représentations à des dates et avec des programmes fixés à l'avance [...], il y a lieu d'encourager ce genre de spectacle tant qu'il donnera satisfaction à la population choletaise ${ }^{69}$ ". Malgré ces conditions, chaque nouveau passage se solde par un échec. Il est impossible de faire reprendre le cycle Baret. En 1935, « bien que la représentation que nous avons donnée il y a deux ans n'ait pas été concluante, nous serions disposés à renouveler cet essai [...] si de son côté, la ville, indépendamment de l'exonération des frais de salle, était disposée à nous consentir une subvention en espèces ${ }^{70}$ ". Nouvel échec. Il y a quelques autres tentatives jusqu'en 1939. Une seule est concluante, avec Les vignes du seigneur de Robert de Flers en février 1936. Cela ne suffit pas à restaurer le cycle Baret à Cholet.

\section{Décadence et concurrence, la fin d'une époque}

La triste fin du cycle Baret à Cholet n'est pas unique. Nous l'avons déjà évoqué, les tournées théâtrales connaissent une évolution positive jusque vers 1930. Après, c'est la descente aux enfers. Le nombre de représentations au théâtre municipal suit chaque année l'état de santé des professionnels du spectacle. La rupture entre 1930 et 1932 est très nette même si, avec dix tournées de passage à Cholet en 1932, cette crise touche moins rapidement

68. Arch. mun. de Cholet, 2R39, 2 mai 1931. Lettre d'un nouvel administrateur général à Gaultier.

69. Arch. mun. de Cholet. Délibérations du conseil municipal, 9 décembre 1933.

70. Arch. mun. de Cholet, 2R39, 6 septembre 1935. Petit à petit on s'achemine vers le système qui prévaut de nos jours : l'achat de spectacles par la municipalité, qui se charge ensuite de les rentabiliser. 
les spectateurs. Ce déclin est-il dû au public qui déserte le théâtre municipal ou aux tournées, plus exigeantes financièrement? Les deux apparemment. Les professionnels du théâtre ont des marges de plus en plus restreintes en raison des charges et cherchent à compenser par une augmentation du prix des places ${ }^{71}$; le public ne suit pas. Entre 1928 et 1930, les prix s'envolent de $30 \%$. Alors que les tournées s'enlisent dans les problèmes financiers, la fréquentation diminue donc avant la fréquence des représentations.

Montré du doigt dès le début de la crise, le cinéma est considéré comme le fossoyeur des tournées théâtrales en détournant sa clientèle. " Il est indéniable que le théâtre subit actuellement une crise imputable au cinéma, ainsi qu'aux fêtes de toutes sortes, crise aggravée par l'apparition du cinéma parlant ${ }^{72}$. " L'expansion du cinéma a sa part de responsabilité dans le délaissement du théâtre, et les chiffres de pleine croissance des cinémas choletais le prouvent. Les adaptations cinématographiques des grands succès théâtraux enfoncent le clou. En 1934 par exemple, La dame de chez Maxim's de Feydeau et Le train de 8 h47 de Courteline, déjà passés au théâtre municipal, font grand bruit dans les salles obscures.

Deux autres éléments s'ajoutent à la crise et amplifient ses effets : l'augmentation du nombre de productions théâtrales amatrices et le théâtre forain. Le registre de la Société des artistes, compositeurs et éditeurs de musique (Sacem) répertorie amateurs et professionnels sans distinctions, pour le paiement des droits d'auteurs. Le graphique qui suit montre que si les productions amateurs ont toujours été majoritaires, elles le sont de plus en plus largement ${ }^{73}$.

\section{Nombre d'œuvres théâtrales jouées à Cholet et déclarées à la Sacem entre 1926 et 1930}

(Source : Arch. mun. de Cholet, 2R32. Registre de la Sacem)

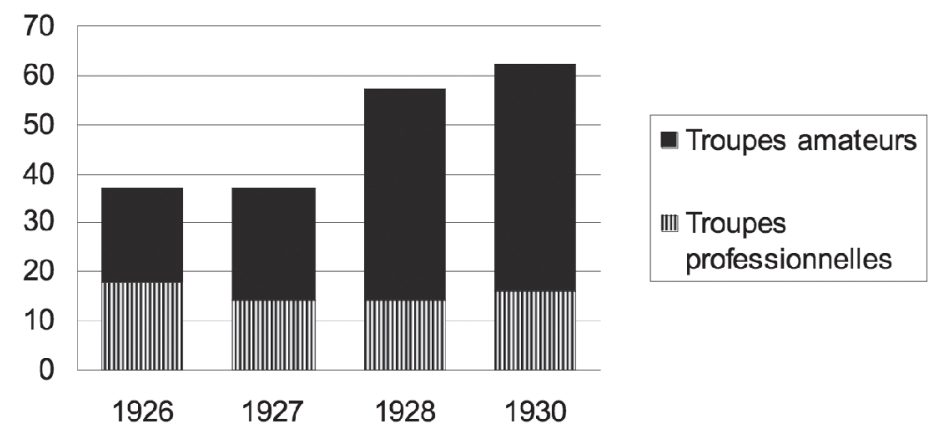

71. Arch. mun. de Cholet, 2R39, 28 août 1926, 19 avril 1928, 13 mars 1930, etc.

72. Arch. mun. de Cholet, Correspondance du maire, 8 juillet 1931 .

73. Arch. mun. de Cholet, 2R32. Registre de la Sacem de 1925 à 1930. 
Le fait que les amateurs offrent plus de rendez-vous théâtraux, à moins cher, contribue sûrement à détourner le public du théâtre municipal. Il faudrait toutefois distinguer deux types d'amateurs : les troupes paroissiales et les troupes laïques. Les premières sont très dynamiques, au réseau large mais cloisonné. Suite à la demande du théâtre du Foyer pour jouer $L a$ Passion à Cholet, Camille Gaultier répond : " Je crois que pour ce genre de spectacle vous avez intérêt à vous adresser aux patronages religieux qui possèdent à Cholet deux salles fort bien agencées ${ }^{74}$. " Les troupes laïques quant à elles sont de plus en plus souvent autorisées à se produire sur la scène du théâtre municipal pour pallier l'absence des tournées. Le maire Alexis Guérineau explique qu'il y a lieu " de tenir compte de la concurrence faite aux artistes professionnels par les troupes amateurs disposant de salles particulières, et plus particulièrement par les patronages où les représentations sont assez fréquentes et très suivies ${ }^{75}$ ".

Difficile à classer, le théâtre forain n'est pas du cirque, mais ne joue pas dans un théâtre. Il s'installe place Travot où il monte un chapiteau, ou dans la salle des fêtes toute récente et propose pendant un temps souvent assez long, une programmation variée équivalente à celle des tournées. Cette résidence dure généralement deux à trois mois pendant la période automnehiver. Les structures sont dures et chauffées, il ne s'agit pas de toiles de tentes montées rapidement. L'attrait principal des théâtres forains, c'est leurs prix. Alignés sur ceux des cinémas, ils sont en moyenne un tiers, voire moitié moins chers que ceux du théâtre municipal. Le théâtre de l'Avenir propose même des places à 10 centimes le jeudi, pour les enfants. Avec cinq séances par semaine, et parfois plus, ces établissements font la différence sur la durée. La programmation est la même que celles des grandes tournées avec toutes les pièces à la mode. " Du comique, du cape et d'épée, du drame..." "résument les frères Delemarre du théâtre forain éponyme. Rominval \& Essers programment des pièces à l'affiche au théâtre municipal à la même période, comme Les Oberlé de René Bazin, non sans quelques soucis. Camille Gaultier est témoin à travers son administration des conflits entre les théâtres forains et les tournées théâtrales. Georges Leduc, qui veut présenter Le mariage de Figaro à Cholet, en janvier 1923, prévient en guise de préambule : « Il faudrait que vous ayez l'obligeance de me donner la certitude que le théâtre forain Delemarre ne sera pas encore installé à cette époque, car si ce théâtre faisait ses débuts aux dates indiquées plus haut pour ma représentation, il serait préférable que je m'abstienne de venir à Cholet avec ma troupe, car cette baraque fait un tort considérable partout où elle passe $[\ldots]$ vu les grosses recettes qu'elle fait, il n'y a donc pas à lutter avec ${ }^{76}$ ! » Même les tournées Baret s'indignent de l'arrivée à Cholet des frères Delemarre : « Nous ne verrions rien à redire s'il n'était question que

74. Arch. mun. de Cholet, 2R35, 17 janvier 1925.

75. Arch. mun. de Cholet. Correspondance du maire, 8 juillet 1931. Lettre du maire au sous-préfet.

76. Arch. mun. de Cholet, 2R35, 3 décembre 1922. 
cet établissement doive séjourner trois mois au moins à Cholet. Ainsi que cela vient de se passer à Saint-Nazaire, ce forain va habituer votre public à des prix de places que ses faibles frais lui permettent de réduire bien audelà de ceux qu'il faut pratiquer au théâtre. Votre population va être sevrée de spectacles pendant de longs mois et il est fort probable que les recettes du théâtre en souffriront encore longtemps après ${ }^{77}$. " L'administrateur s'inquiète pour une représentation prévue cinq semaines après le départ du théâtre forain! Pour rassurer ces impresarios, la municipalité interdit tout spectacle la veille et le jour des représentations au théâtre municipal. Les théâtres forains font évidemment l'objet de critiques bien plus vives à partir des années 1930, quand la concurrence et le mépris cèdent la place aux questions de survie des tournées... Les compagnies se craignent aussi entre elles, c'est pourquoi Georges Leduc exige par exemple, théâtre forain ou pas, cinq jours sans représentations avant son passage et trois jours après.

Comme le cinéma, les amateurs et les forains n'expliquent pas, à eux seuls, la déroute des tournées professionnelles. Le théâtre semble avoir creusé sa propre tombe avant d'y être enseveli par des spectacles moins chers. Franck Evrard explique que « c'est sans doute vers les années 1930 que le fossé idéologique, culturel et esthétique entre le théâtre noble et le théâtre commercial, facile et grand public, qu'il soit tourné vers le social, vers le sérieux (Bernstein) ou vers le divertissement (Courteline, Guitry), se creuse définitivement. Méprisé par les intellectuels, ce théâtre [...] attirait $80 \%$ du public avant-guerre ${ }^{78}$ ». L'érudit local Augustin Jeanneau signe dans le bulletin de la Société des Sciences, Lettres et Beaux-Arts de 1927-1928, un article intéressant sur le théâtre en France. " On parle avec quelque raison chez nous depuis plusieurs années d'une crise du théâtre. En vérité, il n'est pas besoin de beaucoup de clairvoyance pour s'apercevoir du lamentable dénuement de la presque totalité de nos scènes contemporaines ${ }^{79}$. " Ce théâtre de Boulevard, tant courtisé par les foules, a changé. Jeanneau le voit " en pleine décomposition " et rien ne s'arrange avec la crise. Seules $38 \%$ des demandes sont sélectionnées après $1931 \ldots$ pourquoi pas plus ? Le théâtre municipal pourrait ne pas subir les effets de cette crise si les pièces étaient choisies plus largement, ce qui permettrait de retrouver le nombre de spectacles d'avant 1930. Camille Gaultier reçoit suffisamment de demandes, même après 1932, pour maintenir une dizaine de spectacles par an. Le problème réside dans la qualité de ce qui est proposé. Particulièrement après 1934, les pièces à caractère licencieux telles La Fille du bédouin ou Au pays des femmes nues se multiplient et les revues grivoises sont beaucoup plus nombreuses. Musicana spectacles distribue par exemple une brochure avec des photos de femmes aux seins nus et promet " des défilés

77. Arch. mun. de Cholet, 2R35, 26 décembre 1922.

78. EVRARD, Franck, Le théâtre français..., op. cit., p. 27.

79. JEAnneau, Augustin, "L'art du théâtre en France ", Bulletin de la Société des Sciences, Lettres et Beaux-Arts de Cholet, 1927-1928, p. 121. 
suggestifs ${ }^{80}$ ". Jamais, avant cette date, de telles publicités n'avaient été envoyées à Camille Gaultier. Le secrétaire général de la mairie s'intéresse de près à la situation et découpe des articles de journaux sur le sujet. L'un d'eux s'intitule "La grande pitié des théâtres de province ${ }^{81}$ ". Il évoque les taxes multiples qui frappent les tournées, l'augmentation du prix des places, les recettes de plus en plus réduites des impresarios, etc. L'article conclut : "Les scènes seront bientôt envahies par des pièces malsaines, par le cinéma (qui doit rester chez lui) et par les vedettes du music-hall et du bas café-concert. Soutenir le bon théâtre, le vrai, serait donc faire œuvre de salubrité publique. " Le cas de Cholet n'est donc pas isolé, et Camille Gaultier préfère ne rien programmer plutôt que d'accueillir des troupes susceptibles de ternir la réputation de son cher théâtre.

La municipalité porte à bout de bras cet équipement, au prix d'efforts financiers parfois lourds. Dans les comptes, le produit du théâtre est sensiblement le même sur la période allant de 1919 à 1931. Dès 1932, cette ligne budgétaire est en chute dans les recettes de la mairie, jusqu'en 1937 où elle est inexistante. Encore une des manifestations de la crise qui secoue ce milieu.

De nombreux entrepreneurs envisagent tout au long de la période, et particulièrement après 1930, de transformer le théâtre municipal en cinéma. Ce sont à chaque fois de longues lettres adressées au maire pour proposer des tarifications spéciales en fonction du marché déjà présent. La municipalité résiste. "Le déclin du théâtre populaire est rapide face à la revue et surtout au cinéma [...] Les distinctions sociales vont dès lors croissant et le théâtre devient progressivement un spectacle d'élite ${ }^{82}$. " Les augmentations du prix des places vont en ce sens : un siège en galerie coûte 1 franc en 1925 et 3,50 francs en 1930, soit plus du triple, alors que les loges passent de 6 à 14 francs, soit un peu plus du double. Les tournées Baret ne s'y trompent pas et ciblent avec précision leur clientèle en distribuant des brochures et des calendriers. L'administrateur demande à Camille Gaultier " de les faire distribuer de préférence aux professions libérales : avocats, avoués, médecins, dentistes, pharmaciens, rentiers, banquiers, etc. [...] Vous pourriez même en envoyer par la poste aux châtelains des environs ${ }^{83}$ ". Les cafés, à la clientèle plus modeste, sont exclus des circuits de distribution car ils " n'ont aucun intérêt à nous envoyer leurs habitués ". La municipalité est tout de même soucieuse de remplir les places réservées aux classes modestes dans son théâtre car, en comparaison, La Roche-sur-Yon pratique des prix presque deux fois plus élevés ${ }^{84}$. Ces efforts semblent vains. À la fin de notre période, les Choletais ne vibrent plus des éclats de La puce à

80. Arch. mun. de Cholet, 2R36, saison 1934. Prospectus de "Musicana spectacles".

81. Arch. mun. de Cholet, 2R39, coupure de presse jointe au dossier de la saison 1928.

82. Kalifa, Dominique, La Culture de masse en France (1860-1930), Paris, La Découverte, coll. "Repères ", 2001, p. 42.

83. Arch. mun. de Cholet, 2R39, 22 novembre 1936.

84. Arch. mun. de Cholet, 2R32, 12 mai 1920. Correspondance entre le maire de Cholet et le maire de La Roche-sur-Yon. 
l'oreille et autres Folle escapade. Le rideau est tombé sur la belle époque du théâtre municipal.

En une vingtaine d'années, le théâtre municipal de Cholet est l'objet de profonds bouleversements. La gestion de cet équipement culturel est au centre de débats qui en disent long sur la politique hésitante de la ville dans ce domaine et s'achèvent, dans ce cas, par une prise en charge municipale qui doit beaucoup au travail du secrétaire général, Camille Gaultier. Les solutions envisagées sont toutefois de courte durée car le théâtre de Cholet est également témoin, d'une guerre à l'autre, d'autres changements qui s'opèrent à une plus petite échelle. Les professionnels du spectacle connaissent, à partir du début des années 1930, une crise sans précédent, imputable à de nombreux facteurs, et dont les répercussions sont rapidement visibles partout en province. Seule une élite peut s'accrocher au théâtre municipal, mais l'édifice peine à garder son prestige culturel et social. Certes, des applaudissements y résonnent régulièrement jusqu'en 1939 et semblent masquer une déchéance inexorable. Le théâtre populaire vit alors ses dernières heures de gloire, dans la cité du mouchoir comme partout ailleurs.

\section{Camille Gaultier, secrétaire général de la mairie de Cholet, s'est occupé de la gestion du théâtre municipal pen- dant tout l'entre-deux guerres (Collection parti- culière, Ginette Seguin)}

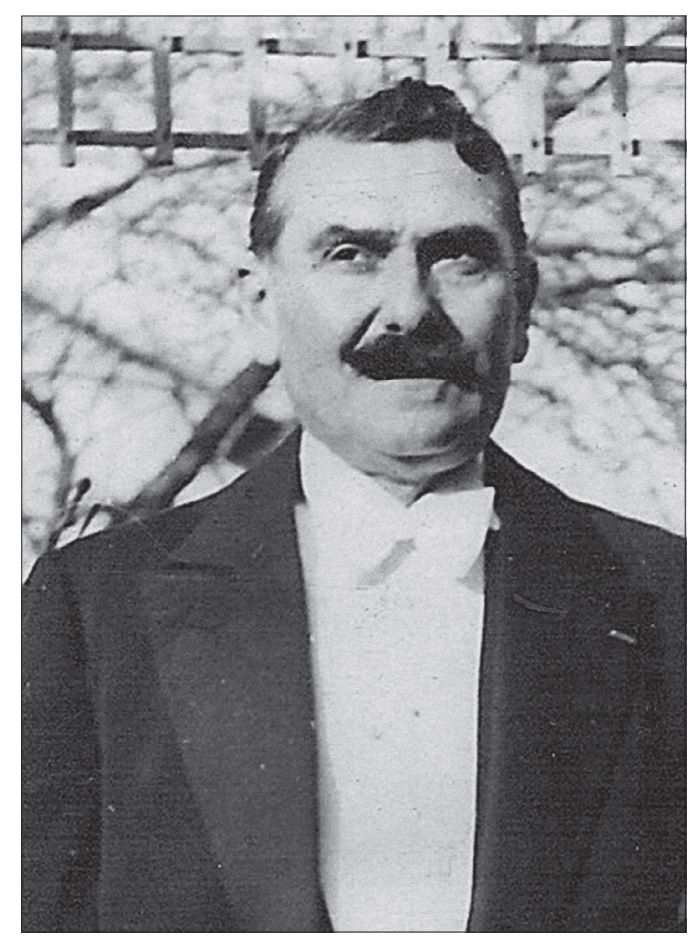


L'affiche de La puce à l'oreille, en représentation à Cholet en décembre 1922 (Archives municipales de Cholet, 2R35, 1922)
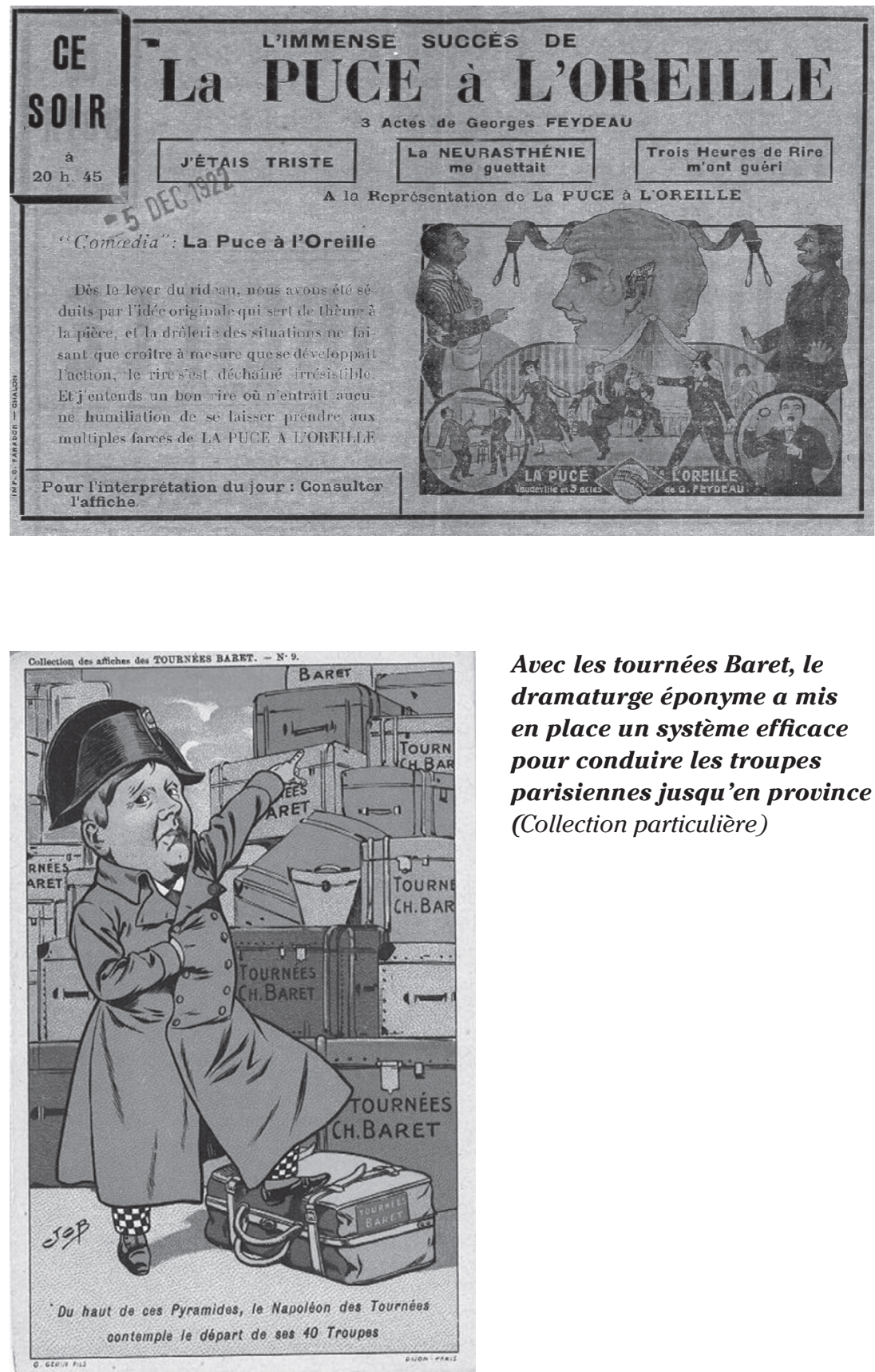

Avec les tournées Baret, le dramaturge éponyme a mis en place un système efficace pour conduire les troupes parisiennes jusqu'en province (Collection particulière) 
Le plafond du théâtre municipal était l'ouvre de l'artiste parisien Audfray. Il n'en est rien resté après l'incendie de 1949 (SOurce : JEANNEAU, Pierre, Le théâtre de Cholet, Cholet, Farré \& Freulon, 1949, p. 16)

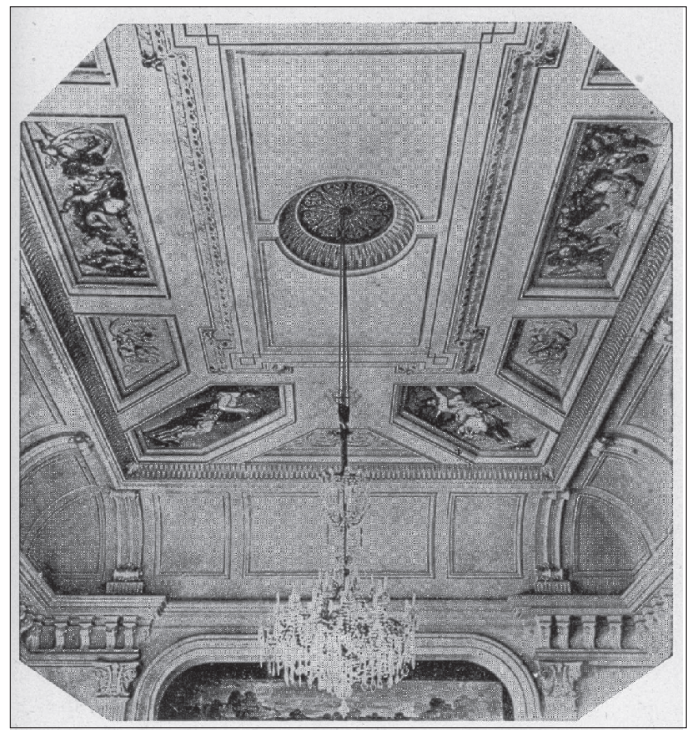

\section{L'affiche de La folle} escapade, en représentation à Cholet en 1923 (Source : Archives municipales de Cholet, 2R36)

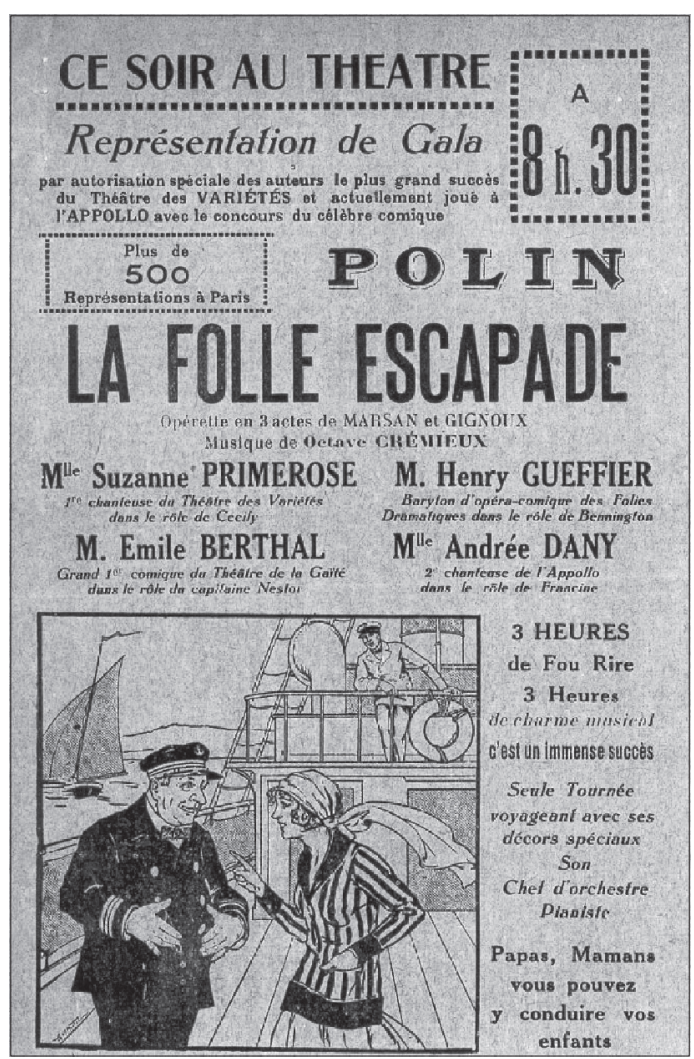


Le théâtre municipal de Cholet aujourd'hui ; l'édifice est toujours au centre de la ville, place Travot (Crédit photographique : Michel Caillard)

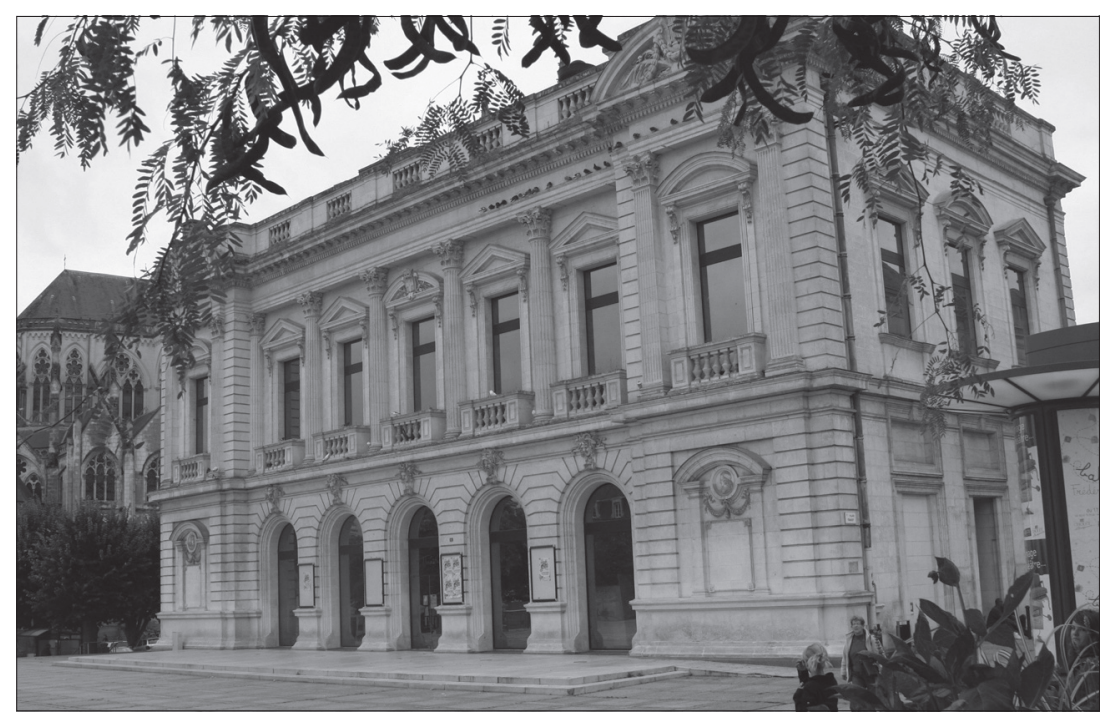

En-tête d'un courrier des tournées Carrington, dans les années 1930 (Source : Archives municipales de Cholet, 2R36)

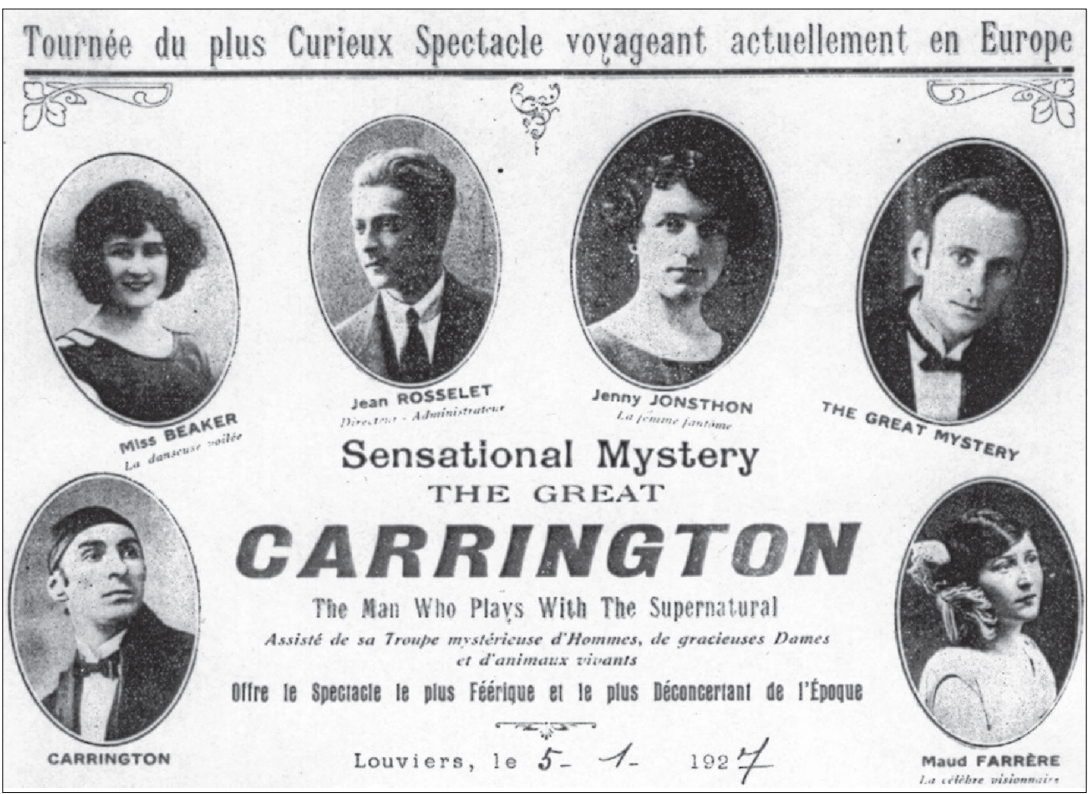


Le théâtre municipal de Cholet pendant l'entre-deux-guerres

Détail de la façade du théâtre municipal. Les sculptures sont de Stanislas Biron, un artiste choletais fort réputé à son époque (Crédit photographique : Michel Caillard)

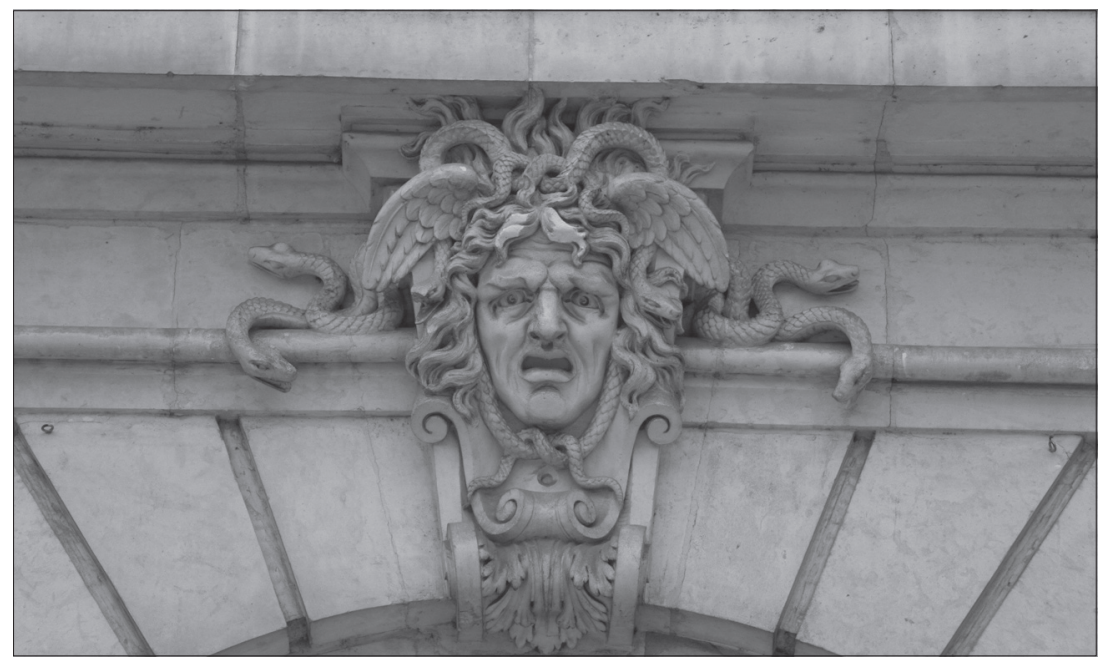




\section{RÉSUMÉ}

Le théâtre de la ville de Cholet trône sur la place principale; c'est un monument important d'histoire et de patrimoine. Il a connu les grandes heures des tournées théâtrales mais aussi les crises de ce milieu artistique, les pleurs et les rires de plusieurs générations. Il a subi les doutes des politiques culturelles de la petite cité de province et l'attention d'une communauté passionnée des arts de la scène. La période de l'entre-deux-guerres est un véritable condensé; et un observatoire de l'organisation des représentations, du répertoire choisi, de la fréquentation ou encore de l'administration de cet équipement culturel de prestige.

\section{ABSTRACT}

The theatre of Cholet is an important historical and heritage monument which occupies a privileged position on the city's main square. It has not only experienced the finest hours of theatrical tours but also artistic crises and the laughter and tears of several generations. It has been a victim of the hesitant cultural policy of this small provincial town and benefited from the patronage of a community passionate about drama. The interwar period encapsulates all of this. It is a suitable period for observing the organisation of performances, the productions chosen, attendance, and indeed the administration of this prestigious cultural monument. 